\title{
Anomalous Vascular Peritoneal Band Causing Small Bowel Obstruction in an Adult
}

\author{
Suzanne Nyakirugumi, Mathenge Nduhiu
}

Nyeri County Referral Hospital, Nyeri, Kenya

Correspondence to: Dr. Suzanne Nyakirugumi; Email:nyuks12@gmail.com

\begin{abstract}
Summary
Peritoneal bands resulting in small bowel obstruction in adults are rare. We present a case study of a 39-year-old male who presented with a 10-day history of signs and symptoms of intestinal obstruction. The patient had no history of abdominal trauma or surgery. Intraoperatively, the small bowel obstruction was caused by a vascularized peritoneal band that had a membrane. The band formed a closed loop and caused the small bowel to herniate and lead to mechanical obstruction. In the band was an anomalous artery that connected the ileocolic artery to the descending branch of the left colic artery. The mainstay for diagnosis is an exploratory laparoscopy or laparotomy. The definitive treatment is transection of the
\end{abstract}

\section{Introduction}

We present a case study of a 39-year-old male with a 10 day history of obstipation resulting from a vascular anomalous peritoneal band. Small bowel obstruction because of peritoneal bands is commonly associated with the pediatric age group. In adults, $60 \%$ of small bowel obstructions are caused by adhesions, most commonly postoperative. Congenital peritoneal bands cause 3\% of intestinal obstruction in adults and children (1). In this case, bowel obstruction was mechanical and the gut failed to propel its contents caudally, leading to build up of material within the lumen. What followed was a vascular compromise because of pressure build up. It may eventually lead to wall ischemia, necrosis and the perforation of gut.

\section{Case Report}

In May 2017, a 39-year-old male patient presented to the Nyeri County Referral Hospital, Kenya, with a 10-day history of progressive abdominal distension and generalized colicky abdominal pain. $\mathrm{He}$ also had postprandial bilious vomiting, on average 5 times daily, and obstipation. The patient had no history of abdominal surgery or trauma, or history of similar symptoms in the band. This is the first reported case in Sub-Saharan Africa.

Keywords: Small bowel obstruction, Congenital bands, Peritoneal bands, Vascular bands, Inferior mesenteric artery, Superior mesenteric artery

Ann Afr Surg. 2020; 17(2):85-87

DOI: http://dx.doi.org/10.4314/aas.v17i2.10

Conflicts of Interest: None

Funding: None

(C) 2020 Author. This work is licensed under the Creative Commons Attribution 4.0 International License. past. He also had no comorbidity. The patient was alert and sick looking on physical examination. His pulse rate was 109 beats per minute, respiratory rate was 22 breaths per minute, temperature $36.8{ }^{\circ} \mathrm{C}$ and blood pressure $128 / 92 \mathrm{mmHg}$. His abdomen was globally distended but moved with respiration. There was generalized tenderness on palpation, and bowel sounds were increased, but no sign of peritoneal irritation. Digital rectal examination revealed an empty rectum. An impression of intestinal obstruction was made. Radiological investigations done on admission were an erect chest $\mathrm{x}$-ray and an erect abdominal $\mathrm{x}$-ray that revealed dilated loops of small bowel with multiple airfluid levels; there was no evidence of extraluminal air. These findings are consistent with small bowel obstruction.

A nasogastric tube inserted drained approximately 1.5 liters of bilious content. The complete blood count was normal. However, he had deranged electrolytes: hypokalemia $2.8 \mathrm{mmol} / \mathrm{L}$, hyponatremia $128 \mathrm{mmol} / \mathrm{L}$ and hypochloremia $88 \mathrm{mmol} / \mathrm{L}$. Serum urea and creatinine were normal.

A decision for exploratory laparotomy was made after correction of electrolytes. The hospital does not have laparoscopic equipment. The patient was infused with 
hypertonic saline and potassium chloride, and blood samples were collected two hours after infusion. The results were ready one and a half hours later and were normal. Four hours after admission the patient was wheeled into theatre.

The abdomen was opened via a midline incision. Distended loops of small bowel were noted. A richly vascularized peritoneal band extending from the left iliac fossa to the mesentery of the cecum was noted (Fig. 1).

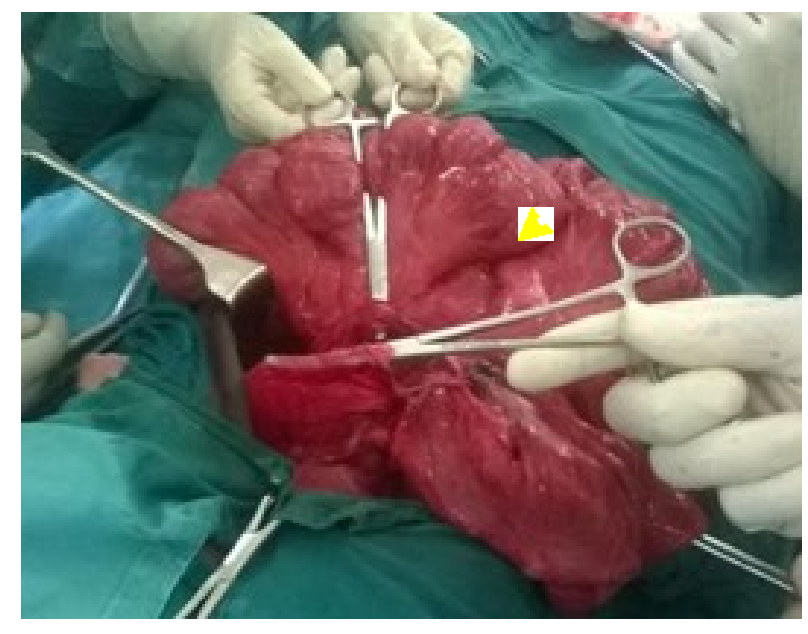

Figure 1: Peritoneal band coming from the left iliac fossa and its membrane held up before transection of the band and the membrane (arrow), below it is small bowel after decompression.

This attachment tethered the appendix, which was midline and anterior to the ileum. The peritoneal band had a membrane overlying the small bowel, and it was proximally attached to the inferior surface of the transverse colon. This formed a pouch on the left upper quadrant where small bowel herniated through and was trapped between the band and its membrane. Approximately $60 \mathrm{~cm}$ of the distal ileum was trapped in the pouch. Within the band was a vessel connecting the ileocolic artery, a branch of the superior mesenteric artery, and the descending branch of the left colic artery, a branch of the inferior mesenteric artery, forming an anastomosis between the two vessels.

The distended small bowel that was trapped was carefully freed from the pouch and manually decompressed. One and a half liters of feculent matter were emptied through a nasogastric tube. The band was then ligated, and the membrane divided along its avascular plane, opening the pouch. The small bowel was inspected and approximately $4 \mathrm{~cm}$ of ischemic bowel was noted about $10 \mathrm{~cm}$ from the ileocecal junction. Resection and an end-to-end, handsewn, single layer anastomosis were done. Anastomosis was done using vicryl $3 / 0$ on a round body needle. The rest of the bowel was normal on inspection. A saline lavage was done. The abdomen was closed in layers and dressed. The patient recovered successfully and was discharged 4 days later.

\section{Discussion}

The origin of anomalous congenital bands is poorly understood. According to Maeda (2), anomalous bands are formed in the embryonic period as remnants of the ventral mesentery (3). Akgur theorizes that these congenital bands are formed around day 26 of intrauterine life during rotation of the gut where the ventral and dorsal mesenteries divide the peritoneal cavity into two halves, the right and left (3). The ventral mesentery disappears as the intestine takes the final position. The dorsal mesentery remains in the posterior part of the abdomen and fuses with the parietal peritoneum. Peritoneal bands result when the ventral mesentery persists. Congenital peritoneal bands, either vascular or avascular, cause $3 \%$ of all intestinal obstructions and often result in small bowel obstruction (1). These bands may be associated with other congenital anomalies that develop during rotation of gut such as malrotation, duodenal atresia, enteric duplication, and sometimes superior mesenteric artery syndrome $(3,4)$. Congenital bands can extend from the ligament of Treitz to the terminal ileum, from the ascending colon to the terminal ileum, and from the right liver lobe to the ascending colon or terminal ileum and in some cases to the bladder $(5,6,7)$. A case is reported where a band originated from the gall bladder to the transverse mesocolon in a 54-year-old adult male, resulting in small bowel obstruction (4).

In our case, the band was from the left iliac fossa to the cecum. Similar to our case, most of the cases reported obstruction on the left side of the abdomen between 15 and $150 \mathrm{~cm}$ from the ileocecal valve with an average of $120 \mathrm{~cm}$ of involved gut, others at the level of the colon $(3,5,8,9)$. These congenital bands can be avascular or vascular; our case was vascular. They may also contain veins and sometimes nerves. Sozen reports a case of intestinal obstruction caused by an anomalous peritoneal band from the antemessenteruim of the terminal ileum to the mesoappendix that contained arteries, veins, and nerve roots (2).

In the reported cases, the blood supply came from branches of the superior mesenteric artery. In our case, however, the band contained an artery that formed an anastomosis between the ileocolic artery, a branch of the 
superior mesenteric artery, and the left colic artery, a branch of the inferior mesenteric artery. This anastomosis between the distal portion of the superior mesenteric artery and the proximal inferior mesenteric artery more distal than anatomically expected made our case unique $(4,5,6)$. Congenital bands are almost always associated with small bowel obstruction, because of the mobile nature of the small bowel.

Most patients require only transection of the band; however, some also require resection and anastomosis due to necrotic gut. Recurrence of intestinal obstruction after transection of the band has not been reported. Diagnosis of congenital bands is made after explorative laparotomy or laparoscopy, which are invasive. There is no known medical treatment. No imaging modality can diagnose congenital bands exclusively as a cause of small bowel obstruction, though radiology can help exclude other major causes of intestinal obstruction. This is the first reported case in Sub-Saharan Africa.

\section{Conclusion}

Peritoneal bands should be considered as a cause of small bowel obstruction in adults with no history of abdominal surgery or trauma. It is important to inspect the band before ligation as it may contain neurovascular structures. The definitive treatment remains surgical, where the band is transected. It is safe to conclude that surgery, either laparotomy or laparoscopy, is both diagnostic and therapeutic.

\section{References}

1. Sozen S, Emir S, Yazar F, et al. Small bowel obstruction due to anomalous congenital peritoneal bands - Case series in adults. Bratisl Lek Listy. 2012; 113(03):186-9.

2. Maeda A, Yokoi S, Kunou T, et al. Intestinal obstruction in the terminal ileum caused by an anomalous congenital vascular band between the mesoappendix and the mesentery: Report of a case. Surg Today. 2004; 34(9):793-5.

3. Akgür F, Tanyel F, Büyükpamukçu N, et al. Anomalous congenital bands causing intestinal obstruction in children. $\mathrm{J}$ Pediatr Surg. 1992; 27(4):471-3.

4. Nayci A, Avlan D, Polat A, et al. Ileal atresia associated with a congenital vascular band anomaly: Observations on pathogenesis. Pediatr Surg Int. 2003; 19(11):742-3.

5. Etensel B, Özkısacık S, Döger F, et al. Anomalous congenital band: A rare cause of intestinal obstruction and failure to thrive. Pediatr Surg Int. 2005; 21(12):1018-20.

6. Habib E, Elhadad A. Occlusion de l'intestin grêle sur bride congénitale chez 16 adultes. Annales de Chirurgie. 2003; 128(2):94-7.

7. Abdelwahed Y, Saber R, Imen B, et al. A case report of small bowel obstruction secondary to a congenital peritoneal band in adult. Int J Surg Case Rep.2017; 30:23-5.

8. Sannananja B, Shah H, Avhad G, et al. Unusual case of an anomalous congenital band causing acute small bowel obstruction in a case of abdominal tuberculosis. J Mahatma Gandhi Inst Med Sci. 2016; 21(1):82-3.

9. KumarA, Ramakrishnan T, Sahu S. Large bowel obstruction by anomalous congenital band. Med J Armed Forces India. 2009; 65(4):378-9. 